\title{
Atypical Lyme Neuroborreliosis, Guillain-Barré Syndrome or Conversion Disorder: Differential Diagnosis of Unusual Neurological Presentations
}

\author{
Tomás Teodoro $^{a} \quad$ Renato Oliveira $^{\mathrm{b}}$ Pedro Afonso ${ }^{c}$ \\ aDepartment of Psychiatry, Lisbon Psychiatric Hospital Center, Lisbon, Portugal; \\ ${ }^{b}$ Department of Neurology, Hospital da Luz - Lisboa, Lisbon, Portugal; ' Department of \\ Psychiatry, Faculty of Medicine, University of Lisbon, Lisbon, Portugal
}

\section{Keywords}

Lyme disease · Neuroborreliosis · Guillain-Barré syndrome · Conversion disorder

\begin{abstract}
Nervous system Lyme disease and Guillain-Barré syndrome are common neurological conditions that may present with unusual symptoms rendering differential diagnosis difficult. We report the case of a 62-year-old woman presenting with asymmetrical tetraparesis and hyporeflexia. Initially the presumed diagnosis of Guillain-Barré syndrome with a possible functional component was suspected and treatment with intravenous immunoglobulin was initiated. Due to partial response to therapy and further test results including positive serologies and cerebrospinal fluid antibodies for Borrelia, the diagnosis of neuroborreliosis was considered. Further exploring revealed the possibility of exposure to ticks although there was no report of typical skin lesions. Daily physical therapy and appropriate antibiotic treatment with parenteral ceftriaxone resulted in significant improvement of motor symptoms and functional status. The patient was discharged with marked functional improvement and indication for further physical rehabilitation.




\section{Case Reports in Neurology}

Case Rep Neurol 2019;11:142-147

DOI: $10.1159 / 000499901$

(C) 2019 The Author(s). Published by S. Karger AG, Base www.karger.com/crn

Teodoro et al.: Lyme Neuroborreliosis, Guillain-Barré Syndrome or Conversion Disorder

\section{Introduction}

Lyme disease is a prevalent tick-borne spirochetal infectious disease with systemic manifestations caused by 1 of 6 species of Borrelia, most frequently B. burgdorferi and B. garinii. The disease may present in three different clinical stages (early localized, early disseminated, late disease). Nervous system involvement occurs only in disseminated disease. Up to $40 \%$ of patients may develop neuroborreliosis [1] although some studies report a lower prevalence of $10-15 \%$ [2]. The classical clinical triad of acute neuroborreliosis includes lymphocytic meningitis, cranial neuropathy, and painful radiculopathy. Peripheral nervous system manifestations include cranial neuropathies, radiculoneuritis, and other forms of peripheral nerve involvement. Neuroborreliosis can rarely present as Guillain-Barré-like syndrome. Diagnostic criteria for nervous system borreliosis include the possibility of exposure to ticks, clinical features associated with neuroborreliosis, and supportive laboratory findings (positive serologies with or without positive antibodies in cerebrospinal fluid [CSF]). Treatment with appropriate antibiotic regimens is usually effective. Full recovery may be expected in patients with early disease although recovery may be slower and incomplete in patients with later stage disease. Guillain-Barré syndrome is a common immune-mediated polyneuropathy associated with multiple precipitant infectious agents, most commonly Campylobacter jejuni. Other agents involved may include cytomegalovirus, Epstein-Barr virus, and human immunodeficiency virus (HIV). The association between Guillain-Barré syndrome and Lyme disease is very uncommon although there are anecdotical reports in the literature [3, 4]. Typical clinical presentation includes progressive ascending bilateral symmetrical limb weakness and decreased tendon reflexes. It is clinically diverse and there are several clinical subtypes, including cranial nerve involvement in Miller-Fisher syndrome. Electrophysiological studies allow for further subtypes to be identified including the axonal and demyelinating forms. Although it is a potentially life-threatening condition, general supportive care and treatment with intravenous immunoglobulin or plasma exchange are generally effective [5]. Conversion disorder is primarily defined by neurological symptoms such as weakness, sensory loss or blackout which, due to internal inconsistency or incongruity with known patterns of disease, are not thought to be caused by a neurological disorder. Despite that, conversion disorder remains a problematic diagnosis. In practice, feigning is very difficult to either disprove or prove, and a psychological explanation cannot always be found [6].

\section{Case Report}

We report the case of a Portuguese 62-year-old woman presenting to the emergency service of a local general hospital with a 2-day history of left-hand and bilateral leg weakness that resulted in two consecutive falls with head trauma. The patient spontaneously reported a temporal association between symptom onset and recent stressful life events. Upon further questioning of her recent history, she reported self-limited diarrhea 3 weeks before. No other symptoms were elicited. She was discharged home after an unremarkable routine blood work and a head computed tomography (CT) without acute findings. However, she returned to the same service the following day after worsening of motor complaints with marked functional impairment and urinary incontinence. On observation, she presented tetraparesis and an 


\section{Case Reports in Neurology}

Case Rep Neurol 2019;11:142-147

DOI: $10.1159 / 000499901$

(C) 2019 The Author(s). Published by S. Karger AG, Basel www.karger.com/crn

Teodoro et al.: Lyme Neuroborreliosis, Guillain-Barré Syndrome or Conversion Disorder

inability to walk independently. The patient was transferred to the neurology department of a central university hospital center for further study. On admission, her neurological examination revealed asymmetric tetraparesis, hyporeflexia, doubtful hemihypesthesia, and left extensor plantar reflex. Weakness was more pronounced on the left side (medical research council [MRC] grade 3/5) than on the right side (MRC grade $4+/ 5$ ). There was distal (MRC grade $3 / 5$ ) more than proximal weakness (MRC grade 4/5) in her lower limbs, also slightly more pronounced on her left leg. Over serial examinations, her motor deficits showed some inconsistencies, seemingly dependent on a variable degree of collaboration and a fluctuating anxious affective state with mild emotional lability. On early observations, Hoover sign was also described. Routine admission tests, including routine blood work, chest X-ray, and electrocardiogram, were unremarkable. Blood work included basic metabolic profile, cell blood count, thyroid and liver studies, vitamin $\mathrm{B}_{1}, \mathrm{~B}_{12}$ and folate levels, lactic acid, erythrocyte sedimentation rate, rapid plasma reagin, human immunodeficiency virus (HIV), venereal disease research laboratory test, antiganglioside antibodies, and angiotensin-converting enzyme levels. Stool cultures were also negative. Her past medical history was irrelevant, including previous drugs or recent vaccination. Considering the possibility of functional neurological symptoms in a patient with anxiety, she was started on sertraline $100 \mathrm{mg}$ and pregabalin $150 \mathrm{mg}$. Her vital signs remained stable throughout her hospital stay.

During the first 4 days of admission, the patient's neurological status remained stable as she underwent daily physical therapy sessions. Head magnetic resonance imaging (MRI) revealed mild white matter T2 and FLAIR hypersignal in relation to chronic microvascular disease. Neuroaxis MRI revealed cervical polyradiculopathy due to cervical canal stenosis and T6-T7 spinal disc herniation without medullary compression. These imaging findings led to a neurosurgery consultation concluding that they did not explain the presenting symptoms and that there was no surgical treatment indication.

After the 5th day of admission, her motor symptoms worsened (MRC grade 2/5 left upper limb, grade $2 / 5$ hip flexion, and grade $2 / 5$ knee flexion and foot plantar flexion) and her upper and lower limbs were areflexic. A lumbar puncture was performed, revealing a normal cell count with an elevated protein level of $0.57 \mathrm{~g} / \mathrm{L}$ (range $0.15-0.45 \mathrm{~g} / \mathrm{L}$ ). CSF glucose, lactate dehydrogenase, white cell count, cytology, Gram stain and culture were normal (Table 1). CSF infectious screen for syphilis, cytomegalovirus, and Epstein-Barr virus was negative. Needle electromyography revealed a subacute motor axonal neuropathy pattern and a right median nerve mononeuropathy. Somatosensory evoked potentials were normal. The CSF albuminocytologic dissociation and electromyography findings suggested the possible diagnosis of Guillain-Barré syndrome. Treatment with a 5-day course of $32 \mathrm{~g} /$ day of intravenous immunoglobulin $(0.4 \mathrm{~g} / \mathrm{kg} /$ day) was initiated with partial improvement of motor function.

Later on, pending laboratory results became available, revealing positive Borrelia serologies (IgM positive and IgG negative). A second lumbar puncture was performed revealing an elevated mononuclear white blood cell count of $20 / \mu \mathrm{L}$ (range $<5 / \mu \mathrm{L}$ ) and normal protein levels. Borrelia IgM antibody was identified with positive confirmatory immunoblot test (Table 2). When inquired the patient reported living in a rural area in England and was on vacation in Portugal. She denied any other recent travel history or noticing any skin changes compatible with erythema migrans. Since the patient remained symptomatic despite previous intravenous immunoglobulin treatment she was started on a 14-day course of ceftriaxone ( $2 \mathrm{~g} /$ day) with significant improvement of the motor function, being able to walk with 


\section{Case Reports in Neurology}

Teodoro et al.: Lyme Neuroborreliosis, Guillain-Barré Syndrome or Conversion Disorder

bilateral support. Despite marked improvement, the patient was discharged with indication for further physical therapy and rehabilitation.

\section{Discussion/Conclusion}

Our patient's presenting symptoms were compatible with acute neurological disease with serious functional impairment. On early examination, objective positive neurological findings included hyporeflexia and an extensor plantar reflex consistent with primary neurological disease. However, she also presented findings that might suggest the diagnosis of conversion disorder including the absence of a typical pattern, the reference to a positive Hoover sign in one of the early evaluations, inconsistent and fluctuating motor deficits, coinciding timeline with stressful life events, and a predominantly anxious basal affective state. The possibility of a conversion disorder should be included in the differential diagnosis of most neurological symptoms. Indeed, before a worsening of her motor symptoms was noticed and further tests were performed, the possible functional nature of the presenting symptoms was seriously considered. Clinical progression with worsening limb weakness and the results of diagnostic tests (lumbar puncture and electromyography) suggested the diagnosis of an acute motor axonal neuropathy variant of Guillain-Barré syndrome. There are case reports of Guillain-Barré syndrome misdiagnosed as a conversion disorder highlighting the importance of considering the clinical heterogeneity of the possible presentations [7]. There was a mild improvement of motor symptoms after a 5-day course of immunoglobulin. Although there was a recent history of diarrhea preceding the symptom onset, none of the most common infectious agents associated with Guillain-Barré syndrome were identified. Surprisingly serologies for Borrelia were revealed to be positive, with further finding of IgM Borrelia antibodies in CSF. The possibility of a Guillain-Barré syndrome due to Borrelia infection should be considered, although this syndrome usually develops as a postinfectious syndrome. In our case, the Borrelia infection appeared to be acute due to its serological profile. These findings in themselves are insufficient for the diagnosis of nervous system Lyme disease since it has been reported that up to $18 \%$ of healthy individuals may have serum antibodies and some show antibody production in their CSF without increased cell count [8]. The second lumbar puncture was performed after a course of immunoglobulin was compatible with neuroborreliosis revealing increased mononuclear white blood cell count and normal protein levels. Clinical findings were, however, highly atypical for nervous system Lyme disease. In favor of a diagnosis of atypical presentation of neuroborreliosis our patient reported the environmental increased possibility of exposure to ticks. Even though she denied recalling a tick bite and associated skin lesions, the literature reports that only $30-40 \%$ of patients with neuroborreliosis report a tick bite upon enquiry and that $50 \%$ do not report skin lesions [1,9]. Although rare, there have been reports of Lyme disease mimicking Guillain-Barré syndrome [10]. There was further clinical improvement after treatment with parenteral ceftriaxone, leading to the patient's discharge.

This case highlights the importance of the differential diagnosis of atypical presentations of neurological disease, including the possibility of functional neurological symptoms. The clinical heterogeneity of certain neurological conditions such as Guillain-Barré syndrome further complicate the establishment of a final diagnosis. In this case, we propose the possible 
diagnosis of an unusual presentation of neuroborreliosis in a patient with a previous presumed diagnosis of Guillain-Barré syndrome.

\section{Statement of Ethics}

Patient consent was obtained.

\section{Disclosure Statement}

The authors have no conflicts of interest to declare.

\section{Funding Sources}

No funding was provided for this article.

\section{Author Contributions}

T.T. developed and wrote the first draft of the manuscript and subsequent revisions; R.O. and P.A. actively collaborated in writing and revising the manuscript.

\section{References}

1 Prasad A, Sankar D. Overdiagnosis and overtreatment of Lyme neuroborreliosis are preventable. Postgrad Med J. 1999 Nov;75(889):650-6.

2 Halperin JJ. Diagnosis and management of Lyme neuroborreliosis. Expert Rev Anti Infect Ther. 2018 Jan;16(1):5-11.

3 Patel K, Shah S, Subedi D. Clinical association: lyme disease and Guillain-Barre syndrome. Am J Emerg Med. 2017 Oct;35(10):1583.e1-2.

4 Samaan M, Venrooy A, Botros C, Puttagunta R. A New Tick Palsy: Case of Guillian-Barre Syndrome Associated with Lyme Disease. Neurology. 2018 Apr;90(15 supplement):P5.136.

5 Willison HJ, Jacobs BC, van Doorn PA. Guillain-Barré syndrome. Lancet. 2016 Aug;388(10045):717-27.

6 Nicholson TR, Stone J, Kanaan RA. Conversion disorder: a problematic diagnosis. J Neurol Neurosurg Psychiatry. 2011 Nov;82(11):1267-73.

7 Edelsohn G. Guillain-Barré misdiagnosed as conversion disorder. Hosp Community Psychiatry. 1982 Sep;33(9):766-7.

8 Mygland A, Skarpaas T, Ljøstad U. Chronic polyneuropathy and Lyme disease. Eur J Neurol. 2006 Nov;13(11):1213-5.

9 Koedel U, Pfister HW. Lyme neuroborreliosis. Curr Opin Infect Dis. 2017 Feb;30(1):101-7.

10 Tyagi N, Maheswaran T, Wimalaratna S. Neuroborreliosis: the Guillain-Barré mimicker. BMJ Case Rep. 2015 Jun 25;2015.pii:bcr2014209080. 
